\title{
Ist Sport für Diabetiker besondes anstrengend?
}

Fragestellung: Strengt körperliche Aktivität postmenopausale Frauen mit Typ-2-Diabetes mehr an als solche ohne Diabetes?

Hintergrund: Typ-2-Diabetes ist mit erhöhter Morbidität und Mortalität assoziiert. Körperliche Aktivität hilft, beides zu reduzieren. Menschen mit Typ-2-Diabetes aber haben häufiger einen deutlich inaktiveren Lebensstil als Gleichaltrige ohne Diabetes. Hierbei ist die Frage, ob der sedative Lebensstil die Ursache für Diabetes ist oder ob er auch die Folge einer Insulinresistenz und Hyperglykämie sein kann? Gerade Letzteres wird verstärkt international diskutiert. Die Zusammenhänge sind aber nicht komplett verstanden. Studien mit prämenopausalen Frauen zeigen, dass der Aufwand für körperliche Aktivität für Frauen mit Diabetes höher ist als für die ohne Diabetes, selbst

\section{Originalie}

Huebschmann AG, Kohrt WM, Herlache L et al. Type 2 diabetes exaggerates exercise effort and impairs exercise performance in older women. BMJ Open Diabetes Research and Care 2015;3: e000124. doi:10.1136/ bmjdrc-2015-000124 wenn sie das gleiche Ziel erreichen. Die aktuelle Studie

nehmer mussten submaximale Leistungen auf einem Fahrradergometer erbringen, wobei 30 Watt Leistung angestrebt wurden oder 35\% des individuell gemessenen maximalen Sauerstoffverbrauchs $\left(\mathrm{VO}_{2}\right.$ max.). Gleichzeitig wurde der Aufwand der Probanden, diese Leistung zu erreichen, mit Hilfe der Borg Scale of Perceived Exertion gemessen. Weiterhin wurden metabolische Parameter, wie Laktat, aber auch Ernährungsverhalten, Taillenumfang und Body Mass Index und das Verhältnis von Muskelzu Fettmasse mit Hilfe des DXA-Scans gemessen. Metabolische Faktoren wie Insulin, Glukose wurden analysiert um Insulinresistenz mittels HOMA zu bestimmen.

Ergebnisse: Es zeigte sich, dass das $\mathrm{VO}_{2}$ max. bei Patientinnen mit Typ-2-Diabetes niedriger ist als bei gesunden Kontrollen. Der Aufwand für die körperliche Aktivität war nicht signifikant größer bei Diabetikerinnen im Verhältnis zu den Kontrollen, aber die gemessenen Laktatspiegel waren bei Diabetes deutlich höher. Der größere Aufwand für körperliche Aktivität war mit höherem Laktat, höherer Herzfrequenz und Bluthochdruck assoziiert.

dieser Zusammenhang be älteren Frauen verhält.

Patienten und Methoden: Postmenopausale Frauen im Alter zwischen 50 und 75 Jahren, mit oder ohne Diabetes (26 vs. 28) wurden in die Studie eingeschlossen. Die Teil-
Schlussfolgerungen: Ältere Typ-2-Diabetikerinnen müssen mehr Aufwand betreiben, um den gleichen sportlichen Effekt zu erreichen wie Kontrollen in der gleichen Altersgruppe. Dies ist wichtig für die Entwicklung von Interventionsprogrammen, da der körperliche Aufwand etwas ist, was durch Training reduziert werden und so Barrieren abbauen kann.

\section{- Kommentar Prof. Dr. med. Peter Schwarz}

\section{Barrieren langsam abtrainieren}

Auf den ersten Blick erscheint die Studie etwas trocken. Denkt man intensiver darüber nach, ergibt sich aber ein logisches Bild. Wenn ich Diabetes habe, bin ich durch meine Physiologie benachteiligt, körperlich aktiv zu sein. Das äußert sich in einem höheren Aufwand (Laktase, Herzfrequenz, Blutdruck) beim Erreichen des gleichen Levels an körperlicher Aktivität. Das wiederrum stellt eine signifikante Barriere dar, weil es von mir als Diabetiker als unangenehm wahrgenommen wird. Damit ergibt sich die logische Konsequenz, dass ich das als Diabetiker vermeide und letztendlich weniger körperlich aktiv bin. Die Autoren beschreiben in ihrer Diskussion sehr deutlich, dass diese Barriere abtrainierbar ist. Und wie ist das möglich? Eben durch körperliche Aktivität. Das, was wir eigentlich schon seit langem wissen und auch lehren, dass das langsame Heranführen an körperliche Aktivität hilft, nachhaltiger körperlich aktiv zu sein, bekommt mit dieser Studie eine physiologische Grundlage. Durch langsam gesteigerte, vermutlich auch häufigere, kurze Phasen körperlicher Aktivität, kann ich die zusätzliche Barriere, die ich als Diabetiker für körperliche Aktivität habe, abbauen. Der Diabetiker hat einen körperlichen Nachteil für Alltags- und sportliche Aktivität, hat aber einen diabetesspezifischen, physiologischen Vorteil. Ein Erfolgsmodell könnte ggf. sein, damit zu beginnen, 5.000, 6.000, 7.000, 8.000, 9.000 und letztendlich 10.000 Schritte am Tag zu laufen und das zu nutzen, um langsam einer sportlichen Betätigung entgegen zu laufen. Die Quintessenz ist, dass die Physiologie für Diabetiker eine zusätzliche Barriere für körperliche Aktivität und Sport ist. Eine langsam steigende Dosis von körperlicher Aktivität hilft diese Barriere zu reduzieren, ansonsten ist der Diabetiker genauso in der Lage, körperlich aktiv zu sein wie der Gesunde, nur profitiert der Diabetiker umso stärker davon.

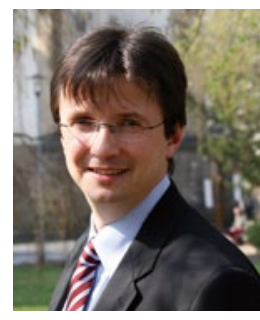

Prof. Dr. med. Peter Schwarz, MBA

Department of Medicine III

Prevention and Care of Diabetes

University of Dresden

Fetscherstr. 74

01307 Dresden

peter.schwarz@uniklinikum-dresden.de 